\title{
Variable Structure Model Reference Adaptive Control for Systems with Unknown High Frequency Gain ${ }^{1}$
}

\author{
Lin Yan, Liu Hsu, Ramon R. Costa, and Fernando Lizarralde \\ Department of Electrical Engineering, COPPE/UFRJ. P.O. Box 68540, Rio de Janeiro, Brazil \\ inyan@ pee.coppe.ufrj.br, iu@ coep.ufrj.br ramon@ coep.ufrj.br fernando@ coep.furj.br
}

\begin{abstract}
In this paper, we consider the design of a variable structure model reference adaptive control (VSMRAC) for plants with relative degree one without the knowledge of the sign of the plant high frequency gain. A switching method for the control signal, based on an appropriate monitoring function, is proposed. As a result, we show that after a finite number of switchings, the tracking error converges to zero at least exponentially. Interestingly enough, if the initial conditions of some states of the closed-loop system are non-zero, we show that at most one switching is needed.
\end{abstract}

Keywords Variable structure control, adaptive control, switching control

\section{INTRODUCTION}

The relaxation of the assumption of high frequency gain sign has long been an attractive topic in control community and can be traced back to the paper by Morse [3]. The most significant advance in this field was due to the work by Nussbaum [4], where an adaptive control law using the so-called Nussbaum gain was used for a one-dimensional linear system. Subsequently, the extension to MRAC for plants with arbitrary relative degree was solved by Mudgett and Morse [5]. Since then, several approaches have been introduced and most of them, however, are based on the Nussbaum-type gain. In [6], a multivariable MRAC using Nussbaum gain was propose. Related work may also be found in [7] in backstepping design. The main disadvantage of Nussbaum-type gain approaches lies in the fact that it lacks robustness to measurement noise. Furthermore, the transient behavior may be unacceptable. Hence, such approaches are of limited practical use [5], [9].

\footnotetext{
${ }^{1}$ Work supported by FAPERJ of Brazil (No. E-26/152.058/2001) and NSF
} of China (No.60174001)
An alternative way is switching. In adaptive control, switching was first introduced by Martennson [8] and then was extended to more general cases by $\mathrm{Fu}$, Barmish, Miller and Davison [9]-[11] with the objective to achieve Lyapunov stability or transient and steady-state performance specifications of tracking error with minimum prior information. The main idea of this kind of control is to design a switching law which may determine among a set of controller candidates when to switch from the current one to the next. It should be pointed out that the robustness to disturbance is still a problem in [8]-[10]. In fact, as shown in [9], [10], if a bounded input or output disturbance exists, the Lyapunov stability may not be retained again and the system states can only tend to some neighborhood of origin that is proportional to the size of the disturbance. In [11], a switching method was proposed so that the tracking error may have an arbitrarily good transient and steady-state performance specifications given by designer in advance even when the plant high frequency gain sign is unknown. However, the price of this solution is that the control signal may be very large. Some other switching methods called hysteresis switching, correction vector approach and adaptive control using multiple models may be found in [12]-[14].

This paper proposes a design method for variable structure model reference adaptive control (VS-MRAC) system without the knowledge of the sign of the plant high frequency gain. The work reported here is based on [1], [2] where a VS-MRAC approach was introduced and shown to have nice transient behavior, disturbance rejection, and performance robustness properties. The objective of this paper is to develop a switching method which 
does not require a priori knowledge of the high frequency gain sign while retaining nice transient performance and input disturbance rejection capability.

In this paper, we first construct a monitoring function and then a switching scheme for the control signal is proposed. We show that under the supervision of the monitoring function, only a finite number of switchings is needed. The tracking error converges to zero at least exponentially. Interestingly enough, if the initial conditions of some states of the closed-loop system are zero, we show that at most one switching is needed. In comparison to those switching approaches presented in [8]-[10], neither the identification of the internal states of closed-loop system nor the Gramian type monitoring function is needed. Furthermore, the input disturbance can be completely rejected without affecting the tracking error performance.

\section{SYSTEM AND ASSUMPTIONS}

Consider the following single input/single output linear time invariant plant

$$
y=G_{p}(s)[u+d]=k_{p}\left(n_{p}(s) / d_{p}(s)\right)[u+d]
$$

where $y$ and $u$ are the system output and input, respectively, $G_{p}(s)$ is the plant transfer function with $d_{p}(s)$ and $n_{p}(s)$ being polynomials of degree $n$ and $m$, respectively, and $d$ is an input disturbance. The objective is to design the control input $u$ so that $y$ tracks as closely as possible the output $y_{M}$ of a stable reference model given by

$$
y_{M}=M(s)[r]=\frac{k_{M}}{d_{M}(s)}[r], k_{M}>0
$$

for any piecewise continuous, uniformly bounded reference signal $r$.

With respect to the controlled plant, we make the following assumptions:

(A1) $G_{p}(s)$ is minimum phase. The parameters of $G_{p}(s)$ are unknown but belong to a known compact set;

(A2) The degree $n$ of $d_{p}(s)$ is a known constant;

(A3) The relative degree $n^{*}=1$;

(A4) The sign of the high frequency gain $k_{p}(\neq 0)$ is unknown;
(A5) The disturbance $d$ satisfies

$$
|d(t)| \leq \bar{d}(t), \forall t \geq 0
$$

where $\bar{d}$ is a known, piece-wise continuous and uniformly bounded function.

In this paper, the control signal is assumed to be of the following form:

$$
u=\hat{\theta}^{T} \omega+u_{v s}
$$

where $u_{v s}$ is a variable structure control term that will be designed to ensure that the tracking error $e:=y$ $-y_{M}$ goes to zero asymptotically, the constant vector $\hat{\theta} \in R^{2 n}$ will be defined below and $\omega$, the regressor vector, is defined as

$$
\omega:=\left[v_{1}^{T}, y, v_{2}^{T}, r\right]^{T} \in R^{2 n}
$$

where $v_{1}$ and $v_{2}$ are generated by input/output filters according to

$$
\begin{gathered}
\dot{v}_{1}=\Lambda v_{1}+g u, \quad \dot{v}_{2}=\Lambda v_{2}+g y, \\
v_{1}(0)=0, v_{2}(0)=0, \Lambda \in R^{(n-1) \times(n-1)}, g \in R^{n-1}
\end{gathered}
$$

where $\Lambda$ is a Hurwitz matrix and $(\Lambda, g)$ is a controllable pair. It is well known [14] that under the above assumptions, there exits a unique constant vector

$$
\theta^{*}=\left[\theta_{1}^{* T}, \theta_{0}^{*}, \theta_{2}^{* T}, k^{*}\right]^{T} \in R^{2 n}
$$

such that, modulo exponentially decaying terms due to initial conditions,

$$
y=G_{p}(s)\left[\theta^{* T} \omega\right]=M(s)[r]=y_{M} .
$$

Since the plant parameters are assumed to be uncertain, the constant vector $\hat{\theta}$ in (2.4) is then defined as

$$
\hat{\theta}:=\left[\hat{\theta}_{1}^{T}, \hat{\theta}_{0}, \hat{\theta}_{2}^{T}, \hat{k}\right]^{T} \in R^{2 n}
$$

which can be obtained from nominal plant and is a rough estimate of $\theta^{*}$. Therefore, the tracking error $e$ can be expressed from (2.1)-(2.9) as

$$
e=M(s) \kappa^{*}\left[\tilde{\theta}^{T} \omega+d_{f}+u_{v s}\right]
$$

where

$$
\begin{gathered}
\tilde{\theta}:=\hat{\theta}-\theta^{*}, \kappa^{*}:=k_{p} / k_{M}=1 / k^{*} \\
d_{f}:=\left(1-d_{1}(s)\right)[d], d_{1}(s):=\hat{\theta}_{1}^{T} \operatorname{adj}(I s-\Lambda) g .
\end{gathered}
$$

\section{VS CONTROL LAW DESIGN}

In this section, we consider the variable structure control law design for plants with $n^{*}=1$. From (2.2), 
$n^{*}=1$ implies that

$$
M(s)=\frac{k_{M}}{\mathrm{~s}+\lambda}
$$

where $\lambda$ is a positive constant. Hence, from (2.10) and (3.1) we have

$$
\dot{e}=-\lambda e+k_{p}\left(\tilde{\theta}^{T} \omega+d_{f}+u_{v s}\right)+\varepsilon
$$

where $\varepsilon$ is a bounded, differentiable and exponentially decaying real function that takes into account all non-zero initial conditions of the internal states of the VS-MRAC system other than those related to $e(0)$. It is important to point out that the discussion before (3.2) is based on the transfer function method which assumes zero initial conditions for all internal states of the system.

The following lemma summarizes the main result when the sign of $k_{p}$ is known:

Lemma 1: Suppose the sign of $k_{p}$ is known. If the VS control signal is defined as

$$
u_{v s}= \begin{cases}-\left(\beta\|\omega\|+\bar{d}_{f}+\Delta\right) \operatorname{sgn}(e), & \text { if } \operatorname{sgn}\left(k_{p}\right)>0 \\ \left(\beta\|\omega\|+\bar{d}_{f}+\Delta\right) \operatorname{sgn}(e), & \text { if } \operatorname{sgn}\left(k_{p}\right)<0\end{cases}
$$

where $\beta$ is an upper bound of $\|\tilde{\theta}\|, \bar{d}_{f}$ is an upper bound of $\left|d_{f}\right|$ and $\Delta$ is an arbitrarily small positive constant, then, the tracking error e converges to zero in some finite time $t=\bar{t}$ and remains zero $\forall t \geq \bar{t}$ as a sliding mode on the surface $e=0$.

Proof. See [1] or [2].

Remark 3.1: Recalling that the parameters of $G_{p}(s)$ are assumed to lie within a known compact set, an upper bound of $\|\tilde{\theta}\|$ can be readily obtained. By taking (2.3) into consideration, an upper bound $\bar{d}_{f}$ satisfying the inequality $\left|d_{f}\right| \leq \bar{d}_{f}$ can also be obtained.

We state the following corollary of Lemma 1, whose proof can be found in [1] or [2].

Corollary 1: The VS-MRAC system is stable if and only if the tracking error $e$ is uniformly bounded.

Since, however, the sign of $k_{p}$ is unknown, we have to redefine the VS control as

$$
u_{v s}(t)=\left\{\begin{array}{r}
u_{v s}^{+}(t):=-\left(\beta\|\omega(t)\|+\bar{d}_{f}(t)+\Delta\right) \operatorname{sgn}(e), \\
\text { if } t \in \mathrm{T}^{+} \\
u_{v s}^{-}(t):=\left(\beta\|\omega(t)\|+\bar{d}_{f}(t)+\Delta\right) \operatorname{sgn}(e), \\
\text { if } t \in \mathrm{T}^{-}
\end{array}\right.
$$

and design a monitoring function to decide when $u_{v s}$ would be switched from $u_{v s}^{+}$to $u_{v s}^{-}$and vice versa, where the sets $\mathrm{T}^{+}$and $\mathrm{T}^{-}$satisfy $\mathrm{T}^{+} \cup \mathrm{T}^{-}=[0, \infty)$ and $\mathrm{T}^{+} \cap \mathrm{T}^{-}=\phi$, and as will be shown in the following analysis, both $\mathrm{T}^{+}$and $\mathrm{T}^{-}$have the form $\left[t_{k}, t_{k+1}\right) \cup \cdots \cup\left[t_{j}, t_{j+1}\right)$. Here, $t_{k}$ or $t_{j}$ denotes the switching time for $u_{v s}$ and will be defined later. The difference between (3.3) and (3.4) is that if the sign of $k_{p}$ is known, we only need one control signal while if the sign of $k_{p}$ is unknown, two control signals, say, $u_{v s}^{+}$ and $u_{v s}^{-}$are needed, where $u_{v s}^{+}$and $u_{v s}^{-}$correspond to $\operatorname{sgn}\left(k_{p}\right)>0$ and $\operatorname{sgn}\left(k_{p}\right)<0$, respectively.

To construct the monitoring function, we consider the following first-order differential equation:

$$
\dot{\xi}=-\lambda \xi+\varepsilon, t \geq \bar{t}_{0}, \xi\left(\bar{t}_{0}\right)=e\left(\bar{t}_{0}\right)
$$

where $\bar{t}_{0} \geq 0$ denotes some initial time for (3.5). Now, if we correctly estimate the sign of $k_{p}$, then by using the Comparison Lemma [16,Th.7] to (3.2) and (3.5), in view of (3.3) and noting that $\xi\left(\bar{t}_{0}\right)=e\left(\bar{t}_{0}\right)$, the following inequality holds for all $t \geq \bar{t}_{0}$ :

$$
|e(t)| \leq|\xi(t)| \text {. }
$$

Remark 3.2: We may also obtain the inequality (3.6) in an alternative way. Let $z:=e-\xi$, then, from (3.2) and (3.5), we have

$$
\dot{z}=-\lambda z+k_{p}\left(\tilde{\theta}^{T} \omega+d_{f}+u_{v s}\right) .
$$

Since $\xi\left(\bar{t}_{0}\right)=e\left(\bar{t}_{0}\right)$, the solution is

$$
\xi=e-k_{p} \int_{\bar{t}_{0}}^{t} \exp [-\lambda(t-\zeta)]\left[\left(\tilde{\theta}^{T} \omega+d_{f}+u_{v s}\right)(\zeta)\right] d \zeta .
$$

The inequality (3.6) hence follows by noting that $u_{v s}$ can completely dominate the term $\tilde{\theta}^{T} \omega+d_{f}$ in the above equation and, according to the assumption, the sign of $k_{p}$ has been correctly estimated.

Our purpose is to construct the monitoring function based on (3.5). We thus consider the solution of (3.5). Since $\varepsilon$ decays exponentially, there exist constants $\delta>0$ and $c>0$ which are independent of any $\bar{t}_{0} \geq 0$, such that 


$$
|\varepsilon(t)| \leq c \exp (-\delta t), t \geq 0 .
$$

Hence, for all $t \geq \bar{t}_{0}$,

$$
\begin{gathered}
|\xi(t)| \leq \exp \left[-\lambda\left(t-\bar{t}_{0}\right)\right]\left|e\left(\bar{t}_{0}\right)\right| \\
+\int_{\bar{t}_{0}}^{t} \exp [-\lambda(t-\zeta)]|\varepsilon(\zeta)| d \zeta \\
\leq \exp \left[-\lambda\left(t-\bar{t}_{0}\right)\right]\left|e\left(\bar{t}_{0}\right)\right|+\left|\frac{c}{\lambda-\delta}\right| \times \\
\times\left|\left\{\exp \left[-\delta\left(t-\bar{t}_{0}\right)\right]-\exp \left[-\lambda\left(t-\bar{t}_{0}\right)\right]\right\} \exp \left(-\delta \bar{t}_{0}\right)\right| \\
\leq \exp \left[-\lambda\left(t-\bar{t}_{0}\right)\right]\left|e\left(\bar{t}_{0}\right)\right| \\
+c_{0} \exp \left[-\delta_{m}\left(t-\bar{t}_{0}\right)\right] \exp \left(-\delta \bar{t}_{0}\right)
\end{gathered}
$$

where

$$
\begin{gathered}
\delta_{m}=\min \{\lambda, \delta\}, \\
c_{0}=2\left|\frac{c}{\lambda-\delta}\right| .
\end{gathered}
$$

Since a less $\delta$ can only make the estimate of $|\varepsilon(t)|$ more conservative, we let $\delta<\lambda$, which implies that

$$
\delta_{m}=\delta \text {. }
$$

Therefore, (3.8) can be rewritten as

$$
\begin{array}{r}
|\xi(t)| \leq \exp \left[-\lambda\left(t-\bar{t}_{0}\right)\right]\left|e\left(\bar{t}_{0}\right)\right|+c_{0} \exp (-\delta t), \\
t \geq \bar{t}_{0} .
\end{array}
$$

Now, we define the monitoring function $\varphi_{k}$ as follows:

$$
\begin{gathered}
\varphi_{k}(t)=\exp \left[-\lambda\left(t-t_{k}\right)\right]\left|e\left(t_{k}\right)\right|+(k+1) \exp \left(-\delta_{k} t\right), \\
t \in\left[t_{k}, t_{k+1}\right), t_{0}:=0, k=0,1, \cdots
\end{gathered}
$$

where $t_{k}$ is the switching time to be defined later, and $\delta_{k}$ is any monotonically decreasing sequence satisfying

$$
\delta_{k} \rightarrow 0 \text { as } k \rightarrow \infty
$$

with $\delta_{0}<\lambda$. The motivation behind the introduction of $\varphi_{k}$ is that $\varepsilon$ is not available for measurement. Comparing (3.12) with (3.13), it is clear that we obtain $\varphi_{k}$ from (3.13) mainly by replacing both $c_{0}$ and $\delta$ by integers $k+1$ and $\delta_{k}$, respectively. Note that the value of $k$ increases as the switching proceeds while $\delta_{k}$ satisfies (3.14) and therefore, we have $\exp (-\delta t)<\exp \left(-\delta_{k} t\right)$ after a finite number of switchings.

Remark 3.3: The term $(k+1)$ in (3.13) may be replaced by any monotonically increasing sequence of $k$.
Recalling that the inequality (3.6) holds if the sign of $k_{p}$ is correctly estimated, it seems natural to use $\xi$ as a benchmark to decide whether a switching of $u_{v s}$ is needed. However, since $\varepsilon$ is not available, we have to use $\varphi_{k}$ to replace $\xi$ and invoke the switching of $\varphi_{k}$. Note that $e$ is absolutely continuous [16], and from (3.13), we always have $\left|e\left(t_{k}\right)\right|<\varphi_{k}\left(t_{k}\right)$ at $t=t_{k}$. Hence, the following definition about the switching time $t_{k}$ for $u_{v s}$ from $u_{v s}^{-}$to $u_{v s}^{+}$(or $u_{v s}^{+}$to $u_{v s}^{-}$) is well-defined:

$$
t_{k}=\left\{\begin{array}{c}
\min \left\{t>t_{k-1}:|e(t)|=\varphi_{k-1}(t)\right\}, \\
\text { if the minimum exists } \\
+\infty, \text { otherwise }
\end{array}\right.
$$

where $k \geq 1$. Fig. 1 illustrates the switching.

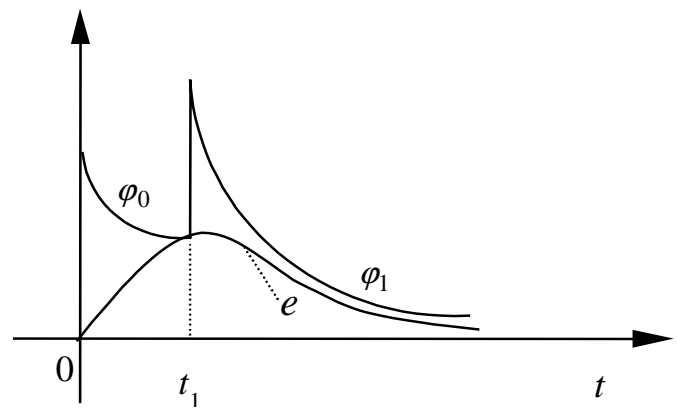

Fig.1. The trajectories of $\varphi_{k}(t)$ and $e(t)$ intersect at $t=t_{1}$ and therefore, a switching of $u_{v s}$ from $u_{v s}^{-}$to $u_{v s}^{+}$(or $u_{v s}^{+}$to $u_{v s}^{-}$) occurs and, at the same time, $\varphi_{k}(t)$ switches from $\varphi_{0}(t)$ to $\varphi_{1}(t)$ according to (3.13). Also note that $t_{0}=0$.

We have the following main result.

Theorem 1: Suppose the VS-MRAC system given by equations (2.1), (3.1) and (2.4) satisfies the assumptions (A1)-(A5). Let the control signal $u_{v s}$ be defined by (3.4) where $\beta$ is an upper bound of $\|\tilde{\theta}\|$, $\bar{d}_{f}$ is an upper bound of $\left|d_{f}\right|$ and $\Delta$ is an arbitrarily small positive constant, and the switching time for $u_{v s}$ from $u_{v s}^{-}$to $u_{v s}^{+}$(or $u_{v s}^{+}$to $u_{v s}^{-}$) be defined by (3.15). Then, the switching will stop after a finite number of switchings and the tracking error e will converge to zero at least exponentially.

Proof. The proof is obtained by contradiction. Suppose $u_{v s}$ switches between $u_{v s}^{+}$and $u_{v s}^{-}$without stopping. Since $c_{0}$ and $\delta$ (see (3.10) and (3.7), 
respectively) are constants, and from (3.4), $u_{v s}$ only has two choices, $u_{v s}^{+}$or $u_{v s}^{-}$, then after a finite number of $k$-th switchings, $u_{v s}$ must have a correct sign, i.e., $u_{v s}=u_{v s}^{+}$if $k_{p}>0$ or $u_{v s}=u_{v s}^{-}$if $k_{p}<0$ and, at the same time,

$$
c_{0}=2\left|\frac{c}{\lambda-\delta}\right|<(k+1), \exp (-\delta t)<\exp \left(-\delta_{k} t\right), \forall t>t_{k}
$$

where $\delta_{k}$ is defined by (3.14). This, together with (3.12) and replacing $\bar{t}_{0}$ by $t_{k}$, implies that

$$
|\xi(t)|<\varphi_{k}(t), \forall t>t_{k}
$$

Since, however, for a correct choice of the sign of $k_{p}$, $e$ satisfies (3.6), the above inequality implies that

$$
|e(t)|<\varphi_{k}(t), \forall t>t_{k} .
$$

Hence, from (3.15), no switching will occur again, a contradiction. Note that $\varphi_{k}$ given by (3.13) is welldefined because as shown in [2], all the closed-loop signals are in $L_{\infty e}$ if the control signal $u_{v s}$ is of the form given by (3.4), or in other words, no finite time escape occurs in the system signals. Now, since $\varphi_{k}$ converges to zero exponentially, (3.18) shows that $e$ will converge to zero at least exponentially. Finally, by invoking the Corollary 1 , the system is stable.

The following corollary shows a more interesting (probably surprising) fact for the relative degree one VS-MRAC system.

Corollary 2: if $\varepsilon=0$, then at most one switching is needed.

Proof. $\varepsilon=0$ implies (3.8) as well as (3.12) can be rewritten as

$$
|\xi(t)| \leq \exp \left[-\lambda\left(t-t_{k}\right)\right]\left|e\left(t_{k}\right)\right|, \forall t \geq t_{k}
$$

where $\bar{t}_{0}$ is replaced by $t_{k}$. Then, (3.13) and (3.19) show that the inequality $|\xi(t)|<\varphi\left(t_{k}\right), \forall t>t_{k}$ holds for any finite $k$ if the sign of $k_{p}$ has been correctly estimated at $t=t_{k}$ which, from (3.6), implies that the following inequality holds also:

$$
|e(t)|<\varphi\left(t_{k}\right) \forall t>t_{k} .
$$

Hence, if we correctly estimate the sign of $k_{p}$ at $t_{0}=0$, by taking (3.15) into consideration, the above inequality shows that no switching occurs; whereas, one switching is enough.

\section{SIMULATION RESULTS}

In this section, an illustrative example is given to show the effectiveness of the switching VS-MRAC scheme. The simulation was done by using a Matlab / Simulink software package.

Example. We consider the following relative degree one plant:

$$
G_{p}(s)=\frac{-(s+1)}{s^{2}-3 s-1}
$$

with different initial conditions:

$$
x(0)=[0,0]^{T}, x(0)=[10,10]^{T}
$$

where $x$ is the state of the controllable canonical form of the plant. Note that in this example, $\operatorname{sgn}\left(k_{p}\right)<0$. The reference model is

$$
M(s)=\frac{1}{s+2} .
$$

Since the parameters of the plant are assumed to be uncertain, $\hat{\theta}$ is obtained according to the nominal plant $\hat{G}_{p}(s)=2(s+0.2) /\left(s^{2}-1\right)$. The parameters of the input/output filters are $\Lambda=-2$ and $g=1$. The monitoring function $\varphi_{k}$ is obtained from (3.14) in which, $\delta_{k}$ is chosen as $\delta_{k}=1 /(k+1)$. The control signal defined by (3.4) is chosen at $t=0$ to be $u_{v s}^{+}$. That is, an incorrect control signal is given at the beginning of the simulation. The reference signal $r$ is a square wave with its amplitude being 1 and frequency being $5 \mathrm{~Hz}$. The disturbance $d(t)=\sin (0.5 t)$. The simulation results under the two different initial conditions are shown in Fig.2 and Fig.3, respectively. Monitoring functions show that for the case of zero initial conditions, the switching number is one while for the case of non-zero initial conditions, three switchings of $u_{v s}$ are observed.

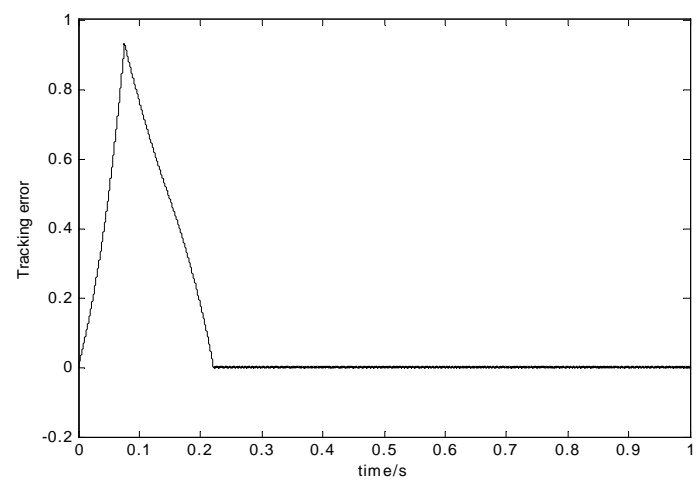

Fig.2-1. Tracking error with zero initial conditions. 


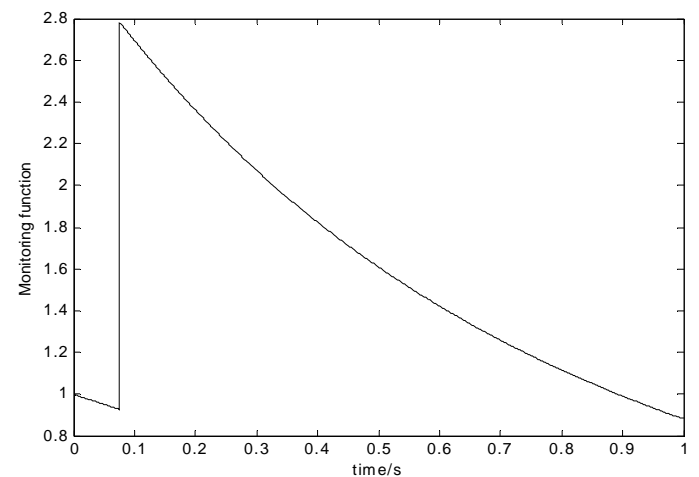

Fig.2-2. Monitoring function with zero initial conditions.

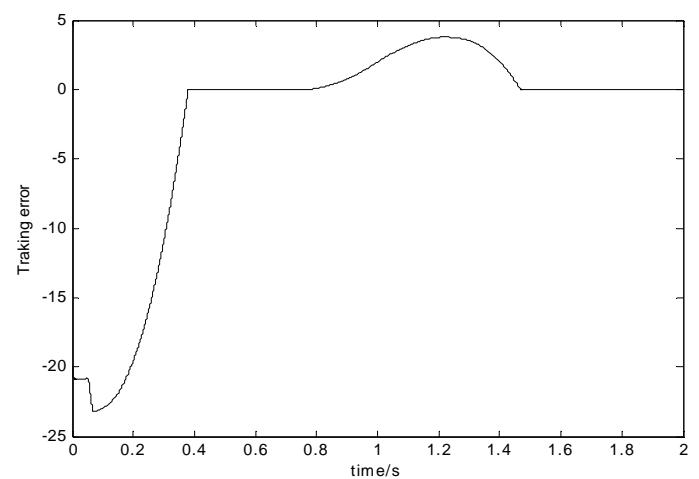

Fig.3-1. Tracking error with non-zero initial conditions.

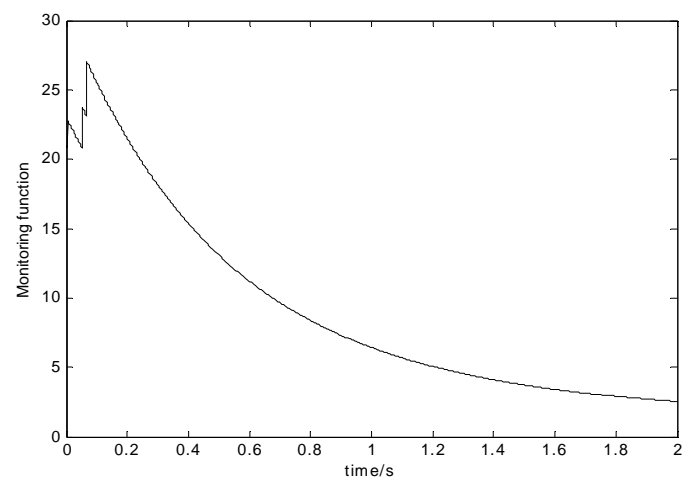

Fig.3-2. Monitoring function with non-zero initial conditions.

\section{CONCLUSION}

In this paper, we have introduced a switching methodology for the controller design of a VS-MRAC system without the knowledge of the high frequency gain sign. The main idea is to construct a monitoring function to supervise the behavior of the tracking error and then, a switching scheme is proposed. We have shown that for plants with relative degree one, our scheme guarantees the tracking error to converge to zero at least exponentially. Another interesting result of the paper is that if some of the initial conditions of the states of the closed-loop system are zero, we have shown that at most one switching is needed. Generalization to plants of higher relative degree is being developed by the authors.

\section{REFERENCES}

[1] Hsu, L. and Costa, R. R., Variable structure model reference adaptive control using only input and output measurementsPart I, Int. J. Control, Vol. 49, No.2: 399-416,1989.

[2] Hsu, L., Lizarralde, F and Araujo, A.D., New results on output-feedback variable structure model-reference adaptive control: Design and stability analysis. IEEE Trans. AC-42, No.3: 42(3): 386-393, 1997.

[3] Morse, A. S., Recent problems in parameter adaptive control, in Proc. CNRS Colloquium on Development and Utilization of Mathematical Models in Automat. Contr., Belle-Isle, France, Sept., 1982, 733-740.

[4] Nussbaum, R. D., Some results on a conjecture in parameter adaptive control, Syst. Contr. Lett., vol.3: 243-246, 1983.

[5] Mudgett, D. R. and Morse, A.S., Adaptive stabilization of linear systems with unknown high frequency gains, IEEE Trans. Automat. Contr., vol. 30: 549-554, 1985.

[6] Imai, A. K., Costa, R. R. and Hsu, L., Multivariable MRAC using Nussbaum gain. In proceedings of 2001 International symposium on adaptive and intelligent systems and control.

[7] Zhang, Y., Wen, C. and Soh, Y. C., Adaptive backstepping control design for systems with unknown high-frequency gain, IEEE Trans. AC-45, No.12: 2350-2354, 2000.

[8] Martensson, B., The order of any stabilizing regulator is sufficient a priori information for adaptive stabilization, Syst. Contr. Lett., vol 6: 85-91, 1985.

[9] Fu, M. and Barmish, B. R., Adaptive stabilization of linear systems via switching control, IEEE Trans. AC-31, No.12: 1097-1103,1986.

[10] Miller, D. E. and Davison, E. J., An adaptive controller which provides Lyapunov stability, IEEE Trans. AC-34, No.6: 599-609, 1989.

[11] Miller, D. E. and Davison, E. J., An adaptive controller which provides an arbitrarily good transient and steady-state response, IEEE Trans. AC-36, No.1: 66-81,1991.

[12] Morse, A. S., Myne, D. Q. and Goodwin, G.C., Application of hysteresis switching algorithm in parameter adaptive control, Proc. $30^{\text {th }}$ IEEE CDC, Brighton.

[13] Lozano-Leal, R., Collado, J. and Mondie, S., Model reference robust adaptive control without a priori knowledge of the high frequency gain, IEEE Trans. AC-35, No.1: 71-78, 1990.

[14] Narendra, K. S., Balakrishnan, J., Adaptive control using multiple models, IEEE Trans. AC-42, No.2: 42(2): 171-187, 1997.

[15] Narendra, K. S, Annaswamy A. M., Stable adaptive control, Prentice Hall, Englewood Cliffs, New Jersey, 1989.

[16] Filippov, A. F., Differential equations with discontinuous right-hand side, Amer. Math. Soc. Translations, vol. 42No.2: 199-231, 1964. 